(c) 2016 IEEE. Personal use of this material is permitted. Permission from IEEE must be obtained for all other uses, in any current or future media, including reprinting/republishing this material for advertising or promotional purposes, creating new collective works, for resale or redistribution to servers or lists, or reuse of any copyrighted component of this work in other works 


\title{
Automatic Differentiation of Normal and Continuous Adventitious Respiratory Sounds Using Ensemble Empirical Mode Decomposition and Instantaneous Frequency
}

\author{
Manuel Lozano*, José Antonio Fiz, and Raimon Jané, Senior Member, IEEE
}

\begin{abstract}
Differentiating normal from adventitious respiratory sounds (RS) is a major challenge in the diagnosis of pulmonary diseases. Particularly, continuous adventitious sounds (CAS) are of clinical interest because they reflect the severity of certain diseases. This study presents a new classifier that automatically distinguishes normal sounds from CAS. It is based on the multi-scale analysis of instantaneous frequency (IF) and envelope (IE) calculated after ensemble empirical mode decomposition (EEMD). These techniques have two major advantages over previous techniques: high temporal resolution is achieved by calculating IF-IE and a priori knowledge of signal characteristics is not required for EEMD. The classifier is based on the fact that the IF dispersion of RS signals markedly decreases when CAS appear in respiratory cycles. Therefore, CAS were detected by using a moving window to calculate the dispersion of IF sequences. The study dataset contained 1494 RS segments extracted from 870 inspiratory cycles recorded from 30 patients with asthma. All cycles and their RS segments were previously classified as containing normal sounds or CAS by a highly experienced physician to obtain a gold standard classification. A support vector machine classifier was trained and tested using an iterative procedure in which the dataset was randomly divided into training $(65 \%)$ and testing $(35 \%)$ sets inside a loop. The SVM classifier was also tested on 4592 simulated CAS cycles. High total accuracy was obtained with both recorded $(94.6 \% \pm 0.3 \%)$ and simulated $(92.8 \% \pm 3.6 \%)$ signals. We conclude that the proposed method is promising for RS analysis and classification.
\end{abstract}

Index Terms-Asthma, continuous adventitious sounds (CAS), ensemble empirical mode decomposition (EEMD), instantaneous frequency (IF), respiratory sounds (RS), wheezes.

\section{INTRODUCTION}

$\mathrm{P}$ ULMONARY auscultation is an essential technique even in cursory physical examinations. Respiratory sounds (RS) contain relevant information about the structure and function of the respiratory system. Therefore, the skills of

This work was supported in part by the Spanish Ministry of Economy and Competitiveness under grant TEC2010-21703-C03-01.

M. Lozano is with the Innovation Group at Health Sciences Research Institute of the Germans Trias i Pujol Foundation (IGTP), Ctra. de Can Ruti, Camí de les escoles, s/n, 08916, Badalona, Spain. He is also with the Institute for Bioengineering of Catalonia (IBEC) (e-mail: mlozano@igtp.cat, mlozano@ibecbarcelona.eu).

J.A. Fiz is with the Pulmonology Service at Germans Trias i Pujol University Hospital, Ctra. de Canyet, s/n, 08916, Badalona, Spain. He is also understanding and interpreting RS are crucial. However, physician subjectivity is a substantial problem in this field and it may lead to an incorrect diagnosis.

RS are generally classified as normal or abnormal [1], [2]. Normal RS originate in healthy lungs, as a consequence of the airflow through the airways during normal breathing. On the other hand, abnormal RS appear in certain pulmonary disorders and they are further classified into discontinuous adventitious sounds and continuous adventitious sounds (CAS). Discontinuous adventitious sounds are explosive and transient sounds that are short in duration but contain a wide range of frequencies. In contrast, CAS typically last more than $100 \mathrm{~ms}$ and are characterized by quasi-periodic waveforms with a fundamental frequency of over $100 \mathrm{~Hz}$ [1], [2].

Identifying abnormal sounds is a key step in the computerized analysis of RS [3]. From a clinical point of view, CAS are important because they are present in various respiratory diseases involving some degree of airway obstruction, such as asthma or COPD [1]. These diseases produce changes in the mechanical properties of the airways, and these changes explain the presence of CAS [4]. Further, there is a relationship between the number of CAS and the severity of airway obstruction in asthmatic patients, severe airway obstruction being related to more CAS [5], [6]. Moreover, the characteristics of CAS, such as mean frequency, duration, intensity, and respiratory phase in which they appear, are also related to the severity of airway obstruction [7], [8]. Therefore, detecting and analyzing CAS may help to assess the severity of obstructive pulmonary diseases, such as asthma, in which measuring the extent of obstruction and its reversibility is important for reaching a correct diagnosis [9]. However, estimating the number of CAS and their characteristics is quite difficult by audition alone. Consequently, there is need for an efficient algorithm to identify and distinguish CAS from

with the IBEC and the Biomedical Research Networking Center in Bioengineering, Biomaterials and Nanomedicine (CIBER-BBN) (e-mail: jafiz@msn.com).

R. Jané is with the Department of Automatic Control (ESAII) at the Universitat Politècnica de Catalunya (UPC). He is also with the IBEC and the CIBER-BBN, Baldiri Reixac, 4, Tower I, 9th floor, 08028, Barcelona, Spain (e-mail: rjane@ibecbarcelona.eu). 
normal RS in a straightforward and reliable way.

Several previous studies have proposed a range of RS classification schemes focused exclusively on distinguishing normal from abnormal RS [10]-[13]. Those studies followed the standard methodology of pattern recognition, which comprises feature extraction, dimensionality reduction, and pattern classification. Features were extracted using either wavelet coefficients [10], morphological complexities (lacunarity, sample entropy, kurtosis, and skewness) [11], multi-scale principal component analysis in Fourier domain [12], or hidden Markov models [13]. The sounds analyzed included many types of adventitious RS, not only CAS. Accordingly, the techniques used did not take into account certain features that are relevant to the accurate identification of CAS, such as temporal features. Further, some other studies followed similar methodologies even though they were focused specifically on wheeze and non-wheeze epoch classification within RS signals. For example, such studies have been based on the combination of Mel frequency cepstral coefficients and Gaussian mixture models [14], [15], or linear analysis using the Fisher discriminant method and the Neyman-Pearson test after extraction of features [16].

In contrast to the aforementioned techniques, the analysis of time-frequency distributions is the most common and straightforward method for CAS identification. The combined analysis of time, frequency, and energy features provides extra information about duration, pitch variation, and magnitude, strengthening the identification of CAS. In this context, many studies have been focused on the identification of wheezingepisodes from spectrograms [17]-[23], by applying temporal and spectral continuity criteria to previously detected peaks. Alternatively, digital image processing techniques have also been applied to the spectrogram for wheeze detection [24], [25], and the scalogram, calculated using the wavelet transform, has been used to classify RS [26]. Recently, more advanced TFDs have been proposed for the detection and analysis of CAS, involving either the combination of the wavelet decomposition with third-order spectral features (instantaneous bispectrum/bicoherence) [27], or the derivation of a temporal-spectral dominance spectrogram from the shorttime Fourier transform [28].

Despite the widespread use of Fourier and wavelet-based techniques, they have some limitations. First, they are nonadaptive techniques, which are limited by the fact that a priori knowledge of the signal characteristics is required for the correct choice of fixed analysis parameters. Second, as is well known, the Fourier transform, like any integration-based transform, is governed by the uncertainty principle, which limits the combined time-frequency resolution and, in turn, the accuracy of TFDs.

Besides the technical aspects, many previous studies used RS signals recorded either at constant airflows or during forced expiratory maneuvers. However, CAS may appear at different airflow levels which, in turn, affect the properties of CAS.

Empirical mode decomposition (EMD) was proposed as an alternative and suitable tool for the analysis of nonlinear and non-stationary signals, such as RS [29]. Since EMD is an adaptive and direct decomposition technique [29], [30], a priori knowledge of the signal characteristics is not required for the choice of analysis parameters. Moreover, EMD allows a signal to be decomposed into a set of components for which the instantaneous frequency (IF) and envelope (IE) can be defined at any point. Therefore, high temporal and spectral resolutions are achieved [31], [32].

In order to take advantage of the EMD-IF properties, we had previously developed a new method for RS analysis based on customized IF and IE estimation by EMD, adapted to RS signal characteristics [33]. In that previous study, we showed that the IF distribution changed when CAS appeared in a respiratory cycle [33]. Nevertheless, we found that the EMD had a mode mixing effect when applied to RS from some inspiratory cycles, which resulted in poor separation of frequency scales.

Building on the results of our previous work, in the present study, we propose a new method to automatically identify CAS and distinguish them from normal RS. The proposed algorithm makes use of ensemble empirical mode decomposition (EEMD) for IF estimation, which improves on the performance of EMD [34]. As we reported in [33], the IF dispersion markedly decreases when CAS appear in respiratory cycles. This property concerns not only wheezes, but any CAS or musical sounds [1] that are characterized by a dominant fundamental frequency. Accordingly, the present study uses this property to detect CAS at a wide range of frequencies. For that purpose, a set of thresholds was applied to the IF dispersion of RS from each inspiratory cycle, delimiting RS segments with a lower IF dispersion. Then, a specific set of features was extracted from the IF and IE sequences to characterize each delimited RS segment within each inspiratory cycle. These features were used to train and test our RS segment classifier, which was a support vector machine (SVM) classifier. Finally, the whole set of inspiratory cycles were classified as containing CAS or only normal RS, depending on whether they contained at least one CAS segment or not. The proposed technique was tested on recorded RS from asthmatic patients, who performed a variable airflow respiratory maneuver to provide CAS at a wide range of airflow levels. This technique was also tested on simulated CAS to analyze the effect of SNR on the performance of the proposed classifier.

\section{MATERIALS AND METHODS}

\section{A. Data Acquisition}

Recorded RS were obtained at the Pulmonary Function Testing Laboratory, Germans Trias i Pujol University Hospital, Badalona, Spain. All recordings were acquired from patients who had previously been diagnosed with asthma in the Pulmonology Service at this hospital. First, lung function was measured by spirometry (Hyp'Air Compact, Medisoft). Then, four piezoelectric contact microphones (TSD108, Biopac Systems, Inc.) were placed on the surface of the patient's back, on each side of the spinal cord, at the base and near the upper lobe of the right/left lung. An additional microphone was placed on the surface of the neck, over the right side of the 
TABLE I

ANTHROPOMETRIC AND SPIROMETRIC CHARACTERISTICS

\begin{tabular}{lc}
\hline Feature/Set & Total \\
\hline Number of subjects & 30 \\
Age $(\mathrm{yr})$ & $45 \pm 14$ \\
BMI $\left(\mathrm{kg} / \mathrm{m}^{2}\right)$ & $25 \pm 4$ \\
FVC $(\%$ of predicted $)$ & $87 \pm 15$ \\
FEV $_{1}(\%$ of predicted $)$ & $80 \pm 17$
\end{tabular}

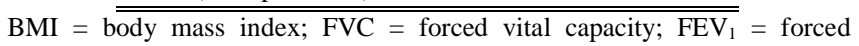
expiratory volume in 1 second.

trachea, at the level of the cricoid cartilage. All sensors were attached to the skin using adhesive rings. The airflow signals were recorded simultaneously with the sound signals, using a pneumotachograph (with an integrated differential pressure transducer, TSD107B, Biopac Systems, Inc.). All signals were sampled at 12500 samples/s, using a 16-bit analogue-to-digital converter (MP150, Biopac Systems, Inc.). After digitalization, the sound signals were band-pass filtered (70-2 $000 \mathrm{~Hz}$ ) and the respiratory phases were automatically detected using the airflow signal as the reference signal.

Patients were asked to perform a respiratory maneuver consisting of progressively varying their airflow levels, ranging from shallow breathing to the deepest breaths they were able to take. We used this maneuver because CAS do not only appear at high airflows, but also at moderate and low airflows. Further, at the beginning and at the end of the respiratory maneuver, patients were asked to hold their breath for a few seconds. Corresponding signal segments were used to evaluate background noise and heart sound interference.

After cycle segmentation, the minimum and the maximum peak flow values of the inspiratory phases delimited the airflow range of each patient. In order to allow comparisons of signals from these different airflow ranges, each one was divided into four proportional intervals or quartiles, from Q1 to Q4, grouping the peak flow values of the corresponding patient in ascending order, from lowest to highest, respectively.

\section{B. Study Dataset}

For this study, RS signals were recorded from 30 asthmatic subjects, whose main anthropometric and spirometric characteristics are summarized in Table I. After cycle segmentation, the study dataset was formed by RS from the inspiratory phase measured with the four microphones on the back, where auscultation is typically performed. We manually selected the inspiratory cycles from the dataset by audiovisual inspection, rejecting those inspiratory cycles containing artifacts (clicks, bursts, or scratches), surrounding noises (background talking or swallowing), or strong heart sound interference (this signal normally being much weaker than RS on the surface of the back). In the end, a total of 870 inspiratory cycles from different airflow quartiles formed the study dataset, including 385 with normal sounds and 485 with CAS (Table II).

A total of 1494 RS segments were extracted from the inspiratory cycles in this dataset. All inspiratory cycles and their corresponding RS segments were manually classified by a highly experienced physician who, by audiovisual
TABLE II

DISTRIBUTION OF THE RECORDED RS

\begin{tabular}{ccccccc}
\hline \hline & \multicolumn{4}{c}{ Inspiratory cycles } & \multicolumn{3}{c}{ RS segments } \\
& Total & Q1 & Q2 & Q3 & Q4 & Total \\
\hline Total & 870 & 163 & 213 & 254 & 240 & 1494 \\
Normal & 385 & 105 & 94 & 109 & 77 & 861 \\
CAS & 485 & 58 & 119 & 145 & 163 & 633 \\
\hline \hline
\end{tabular}

inspection, differentiated those with audible CAS from those with only normal sounds. In this way, we obtained the target classification labels for both the inspiratory cycles and the RS segments. Besides using his personal experience and knowledge in RS analysis, the physician followed the criteria explained in a recent publication about RS [1], and the CORSA guidelines of the European Respiratory Society [2], [3]. According to these guidelines, CAS are defined as musical sounds, heard on inspiration, expiration, or both, with a duration of over $100 \mathrm{~ms}$, and a fundamental frequency above $100 \mathrm{~Hz}$. This definition of CAS includes both wheezes and ronchi. The main difference between them is that rhonchi have lower pitch than wheezes. Based on this definition, different types of CAS were included in the dataset, including some polyphonic CAS. In any case, the goal of this study is not to distinguish between different types of CAS, but to automatically differentiate CAS from normal RS.

\section{Ensemble Empirical Mode Decomposition of RS}

The proposed algorithm for RS classification is based on the fact that RS segments containing CAS have lower IF dispersion than RS segments containing normal sounds in a respiratory cycle, as we reported in [33]. Consequently, the first step of our RS classification scheme is the estimation of the IF of the RS for each inspiratory cycle in the dataset. However, estimating the IF only makes sense for monocomponent or, at least, narrowband signals. As RS are multicomponent signals, we need to decompose them before being able to calculate a physically meaningful IF. In this context, we previously described in [33] that the EMD allows RS signals to be decomposed into a set of components, called intrinsic mode functions (IMFs), for which the IF can be defined at any point. While, ideally, each IMF would contain different frequency components of the RS signal, we found that EMD had a mode mixing effect when applied to some RS signals containing CAS. Due to this mode mixing effect, some frequency components of CAS appeared in different IMFs, leading to poor separation of frequency scales.

Recently, EEMD was proposed as an improvement to EMD to overcome this mode mixing effect [34]. EEMD consists of the iterative application of the original EMD to a signal plus white noise. The idea behind EEMD is that EMD acts as an adaptive dyadic filter bank when applied to white noise, thereby separating the different frequency scales of a signal naturally without any a priori subjective criteria [34]. Moreover, another previous study showed that the computational cost of the EEMD was reduced by replacing white noise by band-limited noise [35], just covering the bandwidth of the signal to be decomposed. 
In the present study, we developed a customized EEMD algorithm adapted to RS signals, for use prior to IF calculations. The basic steps involved in our customized EEMD algorithm are described below:

1) Generate a low-pass filtered white noise signal. Since the fundamental frequency of CAS is typically below $1000 \mathrm{~Hz}$ [1], the cut-off frequency of the low-pass filter was empirically set to $1150 \mathrm{~Hz}$.

2) Add this band-limited noise signal to the RS signal from an inspiratory cycle at an SNR of $0 \mathrm{~dB}$.

3) Decompose the resulting noisy signal into noisy IMFs 1-4 using the original EMD algorithm reported by Rilling and Flandrin [36], [37].

4) Repeat steps one to three 100 times.

5) Calculate the final IMFs as the mean of those resulting from the iteration process.

Fig. 1 illustrates the EEMD method applied to RS from an inspiratory cycle containing a wheeze. IMFs 1-4 were obtained in decreasing order of frequency, with IMF 1 including the highest frequency components in the RS. In this case, the main components of the CAS were within IMF 2. We found that EEMD provided an efficient way to separate the different frequency scales for computerized RS analysis. Moreover, since the frequency range of interest for CAS detection is from $100 \mathrm{~Hz}$ upwards, IMFs 1-4 proved sufficient to cover this range.

\section{Instantaneous Frequency of $R S$}

As defined by Gabor [38], the IF can be calculated from the phase of a complex signal by taking the time derivative. The most practical way to obtain a unique complex signal from a real one is to construct the analytic signal.

In this study, we calculated an analytic signal, $z_{i}(t)$, from each IMF, $s_{i}(t)$, as in (1).

$$
z_{i}(t)=s_{i}(t)+j H\left[s_{i}(t)\right]=a_{i}(t) e^{j \Phi_{i}(t)}
$$

where $H[\cdot]$ is the Hilbert transform, $a_{i}(t)$ is the absolute value of $z_{i}(t)$, and $\Phi_{i}(t)$ is the phase of $z_{i}(t)$. Once we had obtained the analytic signal from each IMF, $I F_{i}(t)$ and $I E_{i}(t)$ could be defined as in (2) and (3), by the phase derivative and envelope of the analytic signal, respectively.

$$
\begin{gathered}
I F_{i}(t)=\frac{1}{2 \pi} \frac{d \Phi_{i}(t)}{d t} \\
I E_{i}(t)=\left|z_{i}(t)\right|=a_{i}(t)
\end{gathered}
$$

A major challenge in calculating the IF for RS is to obtain a physically meaningful frequency, which means not having negative values. In relation to this issue, (2) would only yield true positive values for the IF if $s_{i}(t)$ were to be a monocomponent signal, the phase signal of which is monotonically increasing point-by-point. However, IMFs are not truly monocomponent signals and, therefore, their phase signals could produce unusual negative IF values.

In order to ensure real positive IF values, we developed a smoothing function, which consisted of applying a shapepreserving piecewise cubic interpolation to segments where the phase was not increasing.
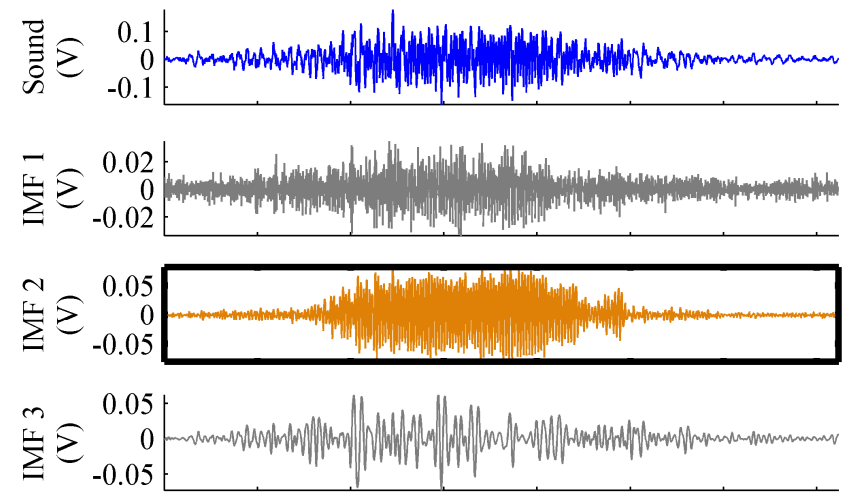

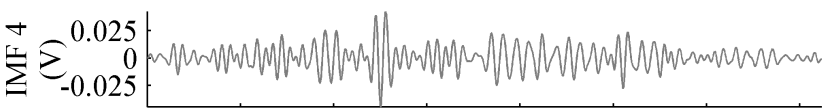

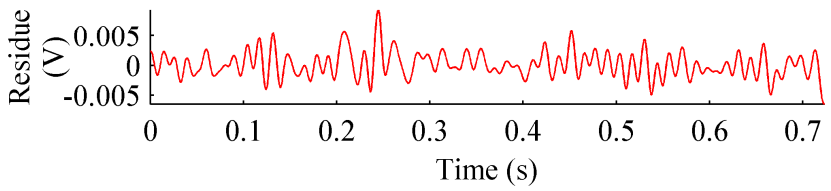

Fig. 1. EEMD applied to RS from an inspiratory cycle containing a wheeze. IMFs 1-4 cover all the frequency range of interest for CAS detection. The major components of the wheeze are within IMF 2 (solid line box).

The complete algorithm to calculate IF and IE comprises the following steps:

1) Calculate IMFs 1-4 by means of EEMD from $\mathrm{RS}$ of each inspiratory cycle.

2) Calculate the analytic signal, $z_{i}(t)$, using the Hilbert transform for each IMF.

3) Obtain the phase signal, $\Phi_{i}(t)$, for each $z_{i}(t)$.

4) Smooth the phase signal using the smoothing function, which prevents IF from taking negative values.

5) Calculate $I F_{i}$ and $I E_{i}$ by differentiating the smoothed $\Phi_{i}(t)$ and taking the absolute value of $z_{i}(t)$, respectively. The time derivative is calculated using a 5-point least squares polynomial approximation [39].

Fig. 2 shows an example of IF sequences obtained from two inspiratory cycles, one containing a wheeze (a) and another with normal RS (b). The IF dispersion was low for as long as the CAS lasted, as shown in IF 2 in Fig. 2 (a). In contrast, IFs from normal RS had uniform dispersions throughout the inspiratory cycle, as shown in Fig. 2 (b).

\section{E. RS Classification}

\section{1) Extraction of Candidate CAS Segments}

Our RS classifier works at two levels: the classification of RS segments within an inspiratory cycle, and the classification of the entire inspiratory cycle. The algorithm extracts RS segments that are candidates for being CAS segments within an inspiratory cycle. Then, feature extraction is applied to each extracted RS segment. These features are used to classify each RS segment, as a normal or CAS segment, using an SVM classifier. Finally, the whole inspiratory cycle is classified as containing CAS or normal sounds, depending on the classification of its RS segments (see Fig. 3). 

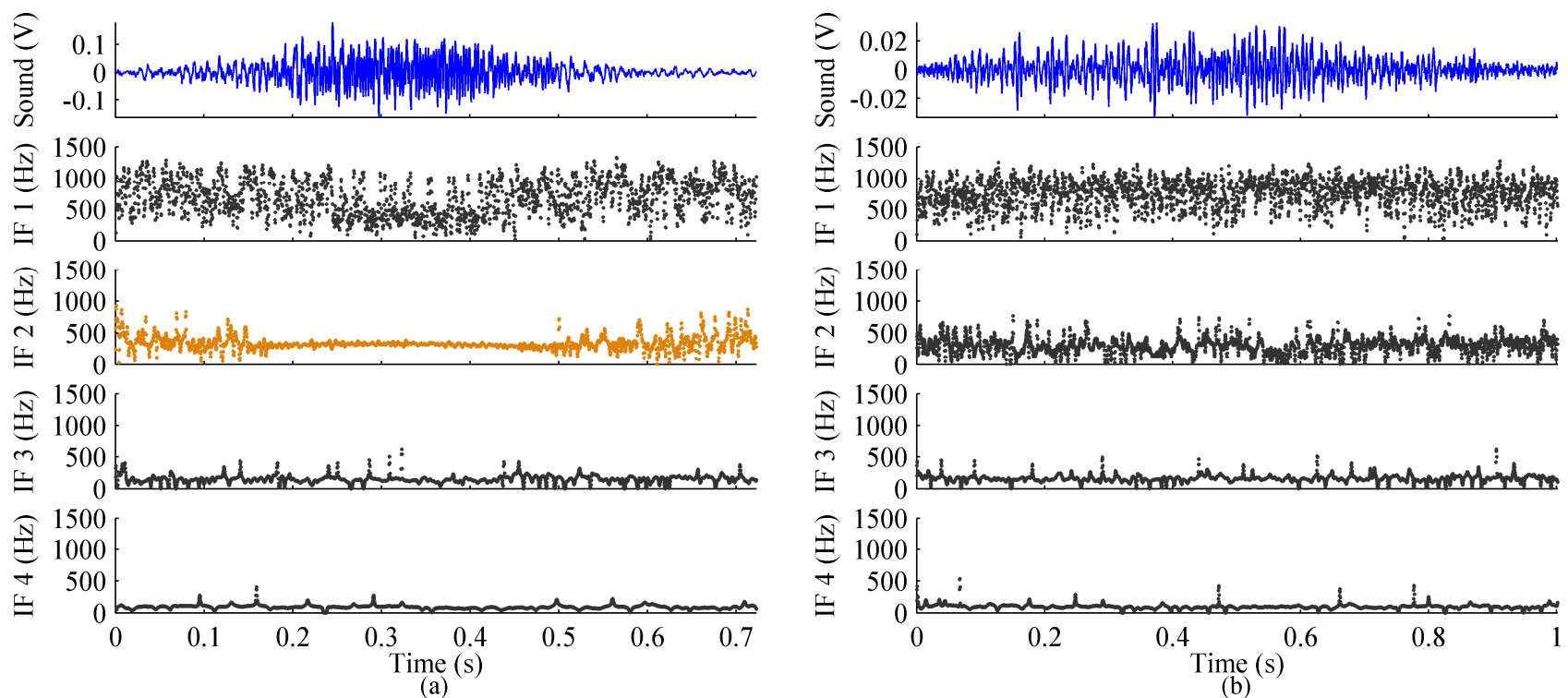

Fig. 2. Instantaneous frequencies (IF 1-IF 4) for IMFs 1-4 from the CAS cycle in Fig. 1 (a), and from one inspiratory cycle with normal RS (b). As shown in (a), a segment with a markedly lower IF dispersion appears within IF 2, and this corresponds to the location of the wheeze. In contrast, IFs from normal RS (b) are highly variable throughout the entire inspiratory cycle.

Given that CAS segments have lower IF dispersion than segments containing normal RS, candidate CAS segments can be detected using a moving window that slides over the entire IF sequences to calculate the IF dispersion $\left(\sigma_{I F i}\right)$. In this study, we used a window length of $40 \mathrm{~ms}$ (125 samples at 3125 samples/second) with $92 \%$ overlap (115 samples). Both parameters were determined empirically. The window length $(40 \mathrm{~ms})$ is less than half of the minimum CAS duration $(100 \mathrm{~ms})$. Shorter window lengths would produce highly variable $\sigma_{I F i}$ sequences, due to unusual spurious IF values. On the other hand, longer window lengths would produce only

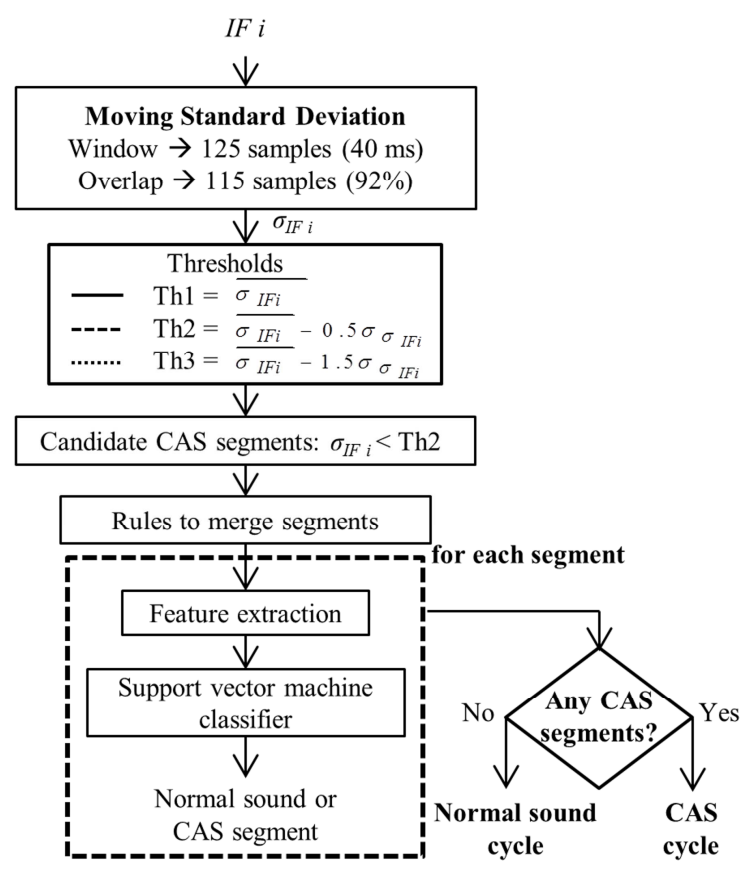

slight variations in the $\sigma_{I F i}$ sequences, making it more difficult to detect CAS segments. The $92 \%$ overlap was chosen to increase the number of data points in the $\sigma_{I F i}$ sequences. If the overlap was small, CAS segments would produce only a few data points with low IF dispersion, whereas, by increasing the overlap between adjacent windows, we obtained more data points improving the accuracy of the detection of CAS segments.

After calculation of the $\sigma_{I F i}$ sequences, a thresholding method was used to detect candidate CAS segments. Thresholds Th1, Th2, and Th3 represent different levels of IF dispersion (Fig. 3) and these were also determined empirically. Threshold Th1 was the mean of an entire $\sigma_{I F i}$ sequence, that is, the mean IF dispersion throughout an entire inspiratory cycle. It was chosen as the reference value to detect CAS segments. The IF dispersion of normal RS segments was expected to be above Th1, whereas the IF dispersion of CAS segments was expected to be below Th1. However, using only Th1 was a weak criterion, which might lead to false CAS segment detections. To avoid false detections, we defined two more restrictive thresholds (Th2 and Th3). Th2 was the upper limit for the IF dispersion of a candidate CAS segment. That is, all segments with an IF dispersion below Th2 were potentially CAS segments. Furthermore, threshold Th3 was used to distinguish those segments with high probability of being CAS segments, due to their very low IF dispersion.

Fig. 4 shows how IF can track not only constant monophonic CAS (a), but also variable monophonic (b) and polyphonic (c) CAS. As shown, the $\sigma_{I F i}$ sequences are below Th2 throughout the presence of CAS.

2) Feature Extraction

Every candidate CAS segment was labeled as one of the

Fig. 3. Flowchart of the proposed RS classification algorithm. 


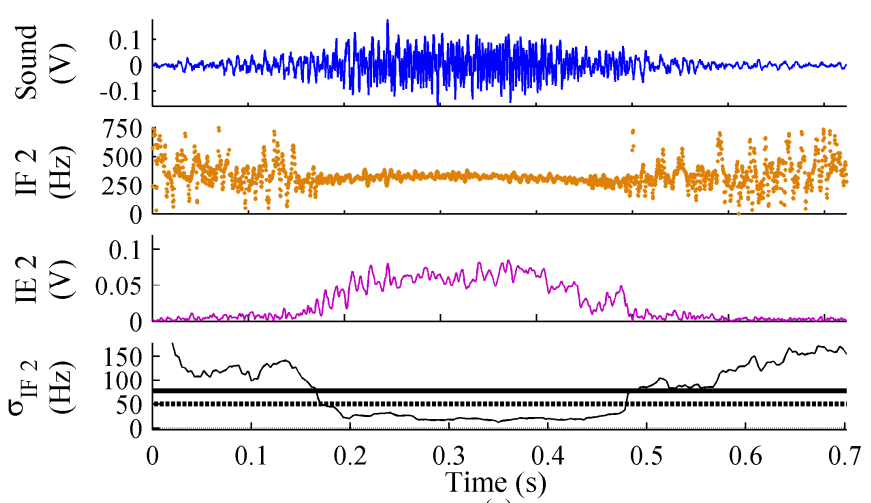

(a)

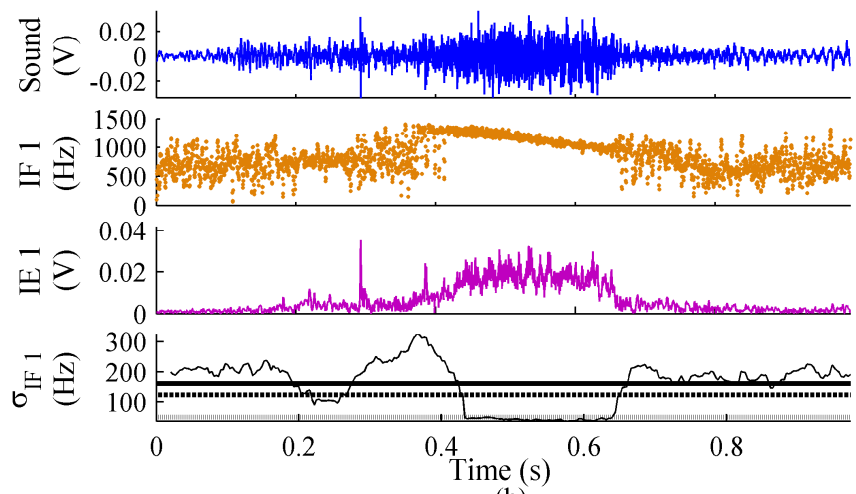

(b)

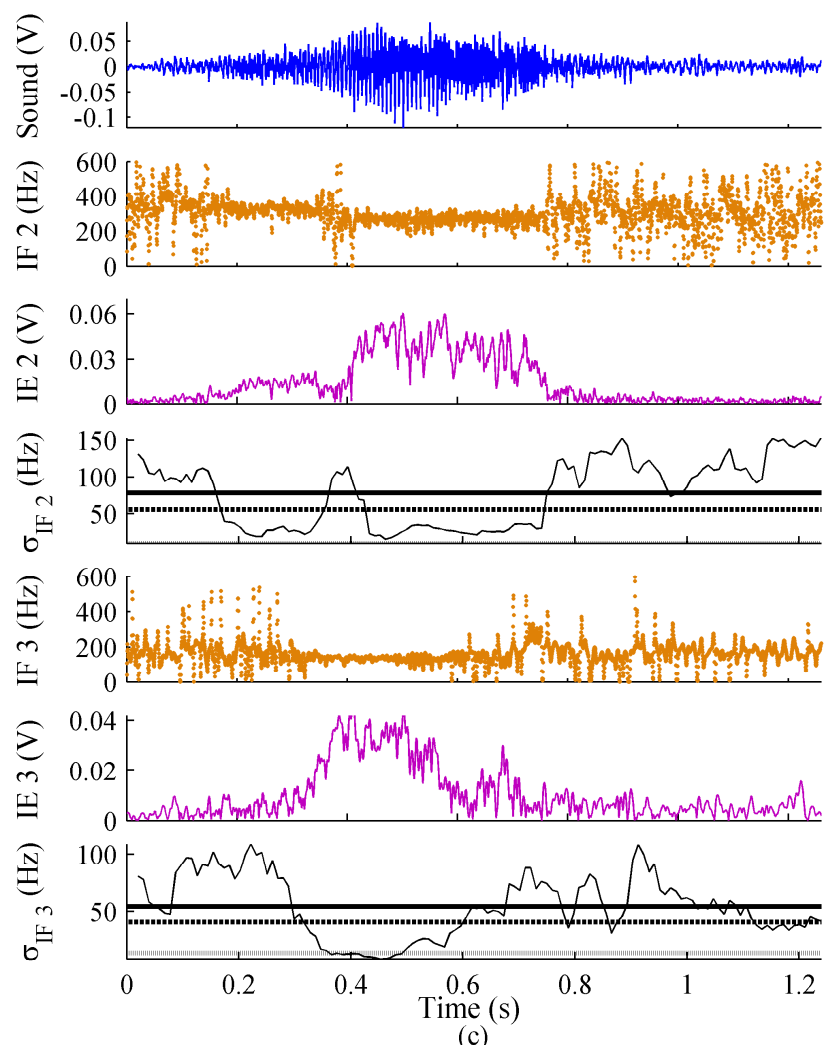

(c)

Fig. 4. Instantaneous frequency (IF i), instantaneous envelope (IE i), and IF dispersion sequence ( $\sigma_{\mathrm{IF}}$ ) from: (a) one CAS cycle containing a constant monophonic wheeze, (b) one CAS cycle containing a variable monophonic wheeze with frequency sweeping, and (c) one CAS cycle containing a polyphonic wheeze, which main components are at mean frequencies of $327 \mathrm{~Hz}$ (IF 2), $266 \mathrm{~Hz}$ (IF 2), and $134 \mathrm{~Hz}$ (IF 3).

following types according to its minimum $\sigma_{I F i}$ and duration,

$d_{1}$ (Fig. 5-a):

Type 1: $\min \left(\sigma_{I F}\right) \leq T h 3, d_{1} \geq 100 \mathrm{~ms}$

Type 2: $\min \left(\sigma_{I F}\right) \leq T h 3, d_{1}<100 \mathrm{~ms}$

Type 3: $\min \left(\sigma_{I F}\right)>$ Th $3, d_{1} \geq 100 \mathrm{~ms}$

Type 4: $\min \left(\sigma_{I F}\right)>T h 3, d_{1}<100 \mathrm{~ms}$

As different RS segments might belong to the same CAS, a grouping algorithm was used to merge RS segments, based on maximum IF dispersion and time elapsed between two adjacent RS segments. Moreover, since the merged segments might have different labels, a priority order was established to
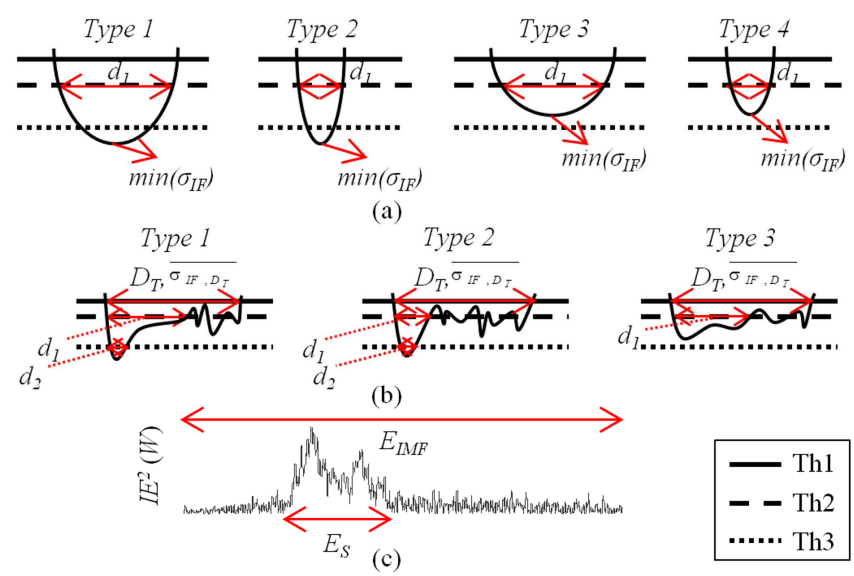

Fig. 5. Characteristic parameters of candidate CAS segments, extracted from the IF dispersion (a), (b), and the instantaneous envelope (IE) (c). set the label for the resulting compound segment, according to the following sequence:

Priority order: Type $1>$ Type $2>$ Type $3>$ Type 4

Each compound segment was labeled as the same type as its highest priority sub-segment.

In the final classification stage, only type 1-3 RS segments were taken into account (Fig. 5-b). Moreover, according to the standard definition of CAS [1]-[3], only RS segments whose total duration $\left(D_{T}\right)$ were greater than or equal to $100 \mathrm{~ms}$ were considered candidate CAS segments. The following features were defined, as in (4), (5), and (6), to characterize the segments (Fig. 5-b, and 5-c).

$$
\begin{gathered}
E R_{1}=100 * \frac{E_{S}}{E_{I M F}}, E R_{2}=100 * \frac{E_{S}}{E_{T}}, E T R=\frac{E R_{2}}{D_{T}} \\
D R=\frac{d_{2}}{d_{1}} \\
Q=\frac{\frac{\sigma_{I F, D_{T}}}{\overline{\sigma_{I F}}}-0.75 \sigma_{\sigma_{I F}}}{}
\end{gathered}
$$

where $E_{I M F}$ is the sum of all terms of the squared IE of an IMF, $E_{S}$ is the sub-interval of $E_{I M F}$ corresponding to the segment location, $E_{T}$ is the sum of $E_{I M F 1}, E_{I M F 2}, E_{I M F 3}$, and $E_{I M F 4}$, and $\overline{\sigma_{I F, D_{T}}}$ is the mean IF dispersion over the length of the segment. These five features correspond to measurements that depend either on the energy $\left(E R_{1}, E R_{2}\right.$, and $\left.E T R\right)$ or on the IF dispersion ( $D R$ and $Q$ ) of RS segments. 
3) RS Segment Classification using Support Vector Machines

The proposed SVM classifier works at the segment level of our classification scheme (Fig. 3). That is, the input to the SVM classifier was a numerical data vector containing features $E R_{l}$, $E R_{2}, E T R, D R$, and $Q$, and the IMF number (from 1 to 4 ) of an RS segment. In order to train and test our SVM classifier, we used an iterative procedure, as illustrated in Fig. 6.

First, the inspiratory cycles from the full dataset were divided into two subsets, one for training (559 cycles) and another for testing (311 cycles). Due to the high betweensubject variability of the RS, the subsets were formed independently and hence included inspiratory cycles from different asthmatic patients. Then, $921 \mathrm{RS}$ segments extracted from the training inspiratory cycles were used to find the optimum parameters for our SVM classifier (the regularization parameter, $\mathrm{C}$, and the kernel parameter, $\sigma$ ). For that purpose, we used a $5 \times 5$ grid with increasing sequences of $\mathrm{C}$ (from 1 to 3 ) and $\sigma$ (from 0.1 to 1 ). Each combination of parameters was the starting point for finding a local minimum of the 10 -fold cross-validation loss function. The parameters which produced the lowest cross-validation loss were chosen as the optimum parameters $\left(\mathrm{C}_{\mathrm{opt}}=2.72\right.$ and $\left.\sigma_{\mathrm{opt}}=1.61\right)$.

Having found the optimum SVM parameters, we employed them to train an SVM classifier using the RS segments from the training inspiratory cycles. The resulting SVM classifier was validated using the RS segments from both training and testing inspiratory cycles and the target labels from the manual RS segment classification. The performance of the SVM classifier was evaluated in terms of accuracy, sensitivity, specificity, and positive predictive value.

Since the results obtained might depend on the initial grouping of the inspiratory cycles, we repeated the training and testing steps using different partitions of the data. For that purpose, the inspiratory cycles from the dataset were randomly

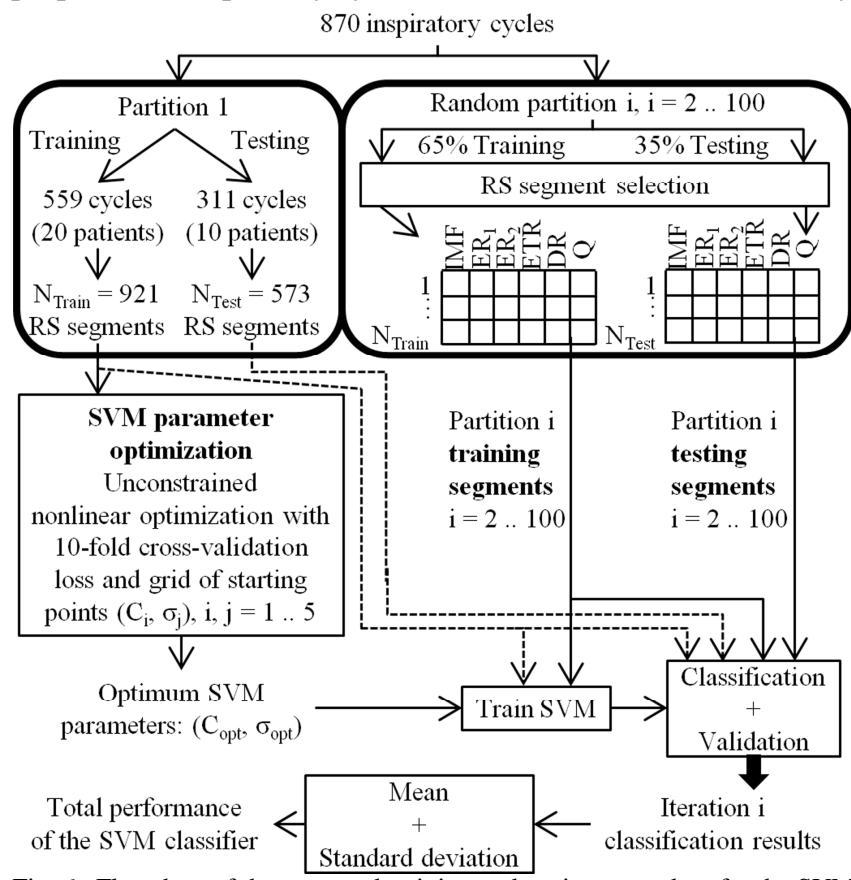

Fig. 6. Flowchart of the proposed training and testing procedure for the SVM classifier. divided into training (65\%) and testing (35\%) sets inside a loop. The random partitions were done in such a way that each subset contained a proportionate share of the normal and CAS Q1-Q4 inspiratory cycles from the full dataset (Table II). After each random partition, the RS segments from the resulting training and testing inspiratory cycles were used to train and test an SVM classifier with the optimum parameters $\left(\mathrm{C}_{\mathrm{opt}}\right.$ and $\sigma_{\text {opt }}$ ). The total performance of the SVM classifier was calculated as the mean and the standard deviation of the classification results of each iteration.

4) Inspiratory Cycle Classification

Considering the RS segment classification obtained in each iteration of the previous stage, each entire inspiratory cycle was classified as containing CAS if any of its RS segments was classified as a CAS segment. Otherwise, the entire inspiratory cycle was classified as containing normal sounds.

The performance of the SVM classifier was also evaluated at cycle level, by comparing the inspiratory cycle classification obtained with the classifier to the manual inspiratory cycle classification.

We selected the SVM with the best performance, among all iterations, for the classification of simulated CAS cycles in the next Section II.F.

\section{F. Simulation of CAS Cycles}

This section describes a procedure to study the effect of duration and SNR of simulated CAS on the performance of the classifier. For that purpose, the classifier was tested on 4592 simulated CAS cycles. The procedure to obtain the simulated CAS cycles was as follows:

1) Eight CAS segments were manually extracted from different inspiratory cycles with CAS from the study dataset.

2) Either time contraction or dilation was applied to those eight CAS segments in order to obtain 41 new CAS segments, $n_{i}(t)$, thus covering time durations from $100 \mathrm{~ms}$ to $500 \mathrm{~ms}$, in increments of $10 \mathrm{~ms}$.

3) Each one of those 41 CAS segments was added to 7 inspiratory cycles with normal sounds, $s_{k}(t)$, as in (7), at $16 \mathrm{SNRs}, S N R_{j}$, from $-5 \mathrm{~dB}$ to $10 \mathrm{~dB}$, in increments of 1 dB. As a result, a total of 656 simulated CAS cycles, $w_{i j k}(t)$, were obtained for each original normal sound cycle, $s_{k}(t)$.

$$
w_{i j k}(t)=s_{k}(t)+n_{i j k}(t), i=1 . .41, j=1 . .16, k=1 . .7
$$

In (7), $i$ is the CAS segment subscript, $j$ is the SNR subscript, and $k$ is the subscript for the inspiratory cycle with normal sounds. In this study, the SNR was defined as in (8). 


$$
\begin{gathered}
S N R_{j}=10 \log \left(\frac{P_{n_{j k}}}{P_{S_{k}}}\right) \rightarrow P_{n_{j k}}=P_{S_{k}} 10^{\frac{S N R_{j}}{10}} \\
n_{i j k}(t)=\sqrt{\frac{P_{n_{j k}}}{P_{n_{i}}}} n_{i}(t)
\end{gathered}
$$

where $P_{S_{k}}(t)$ is the power of $s_{k}(t), n_{i j k}(t)$ is the $n_{i}(t)$ CAS segment added to the $s_{k}(t)$ inspiratory cycle at $S N R_{j}, P_{n_{j k}}(t)$ is the power of $n_{i j k}(t)$, and $P_{n_{i}}$ is the power of $n_{i}(t)$.

In this way, we obtained a set of simulated CAS cycles that allowed us to explore the effectiveness of the proposed algorithm in terms of two basic parameters of CAS: duration and intensity. The influence of these parameters on each simulated CAS cycle was calculated as the percentage increase in mean power with respect to the original cycle with normal sounds, $s_{k}(t)$, given by expression (10).

$$
\Delta P_{i j k}=100 \frac{P_{w_{i j k}}}{P_{S_{k}}}
$$

This new parameter allowed us to analyze the classification results of the simulated CAS cycles for different ranges of power increase.

\section{RESULTS}

\section{A. Recorded RS Classification}

The performance of the SVM classifier at segment level, obtained after the training and testing described in Section II.E.3, is summarized in Table III. As shown, high accuracy $(94.0 \% \pm 0.8 \%)$ and sensitivity $(92.8 \% \pm 1.7 \%)$ were achieved with the test set for classifying recorded RS segments.

The performance of the SVM classifier at cycle level is reported in Table IV. As shown, our classifier achieved high total accuracy $(94.6 \% \pm 0.3 \%)$ and sensitivity $(94.2 \% \pm 0.4 \%)$ for classifying recorded RS from inspiratory cycles as normal sounds or CAS. Among all iterations, the best SVM classifier had an accuracy of $95.1 \%$, a sensitivity of $94.2 \%$, a specificity of $96.1 \%$, and a positive predictive value of $96.8 \%$, at cycle level. That SVM classifier was used to obtain the classification
TABLE III

CLASSIFICATION OF RECORDED RS SEGMENTS

\begin{tabular}{lccc}
\hline \hline Parameter/Set & Total & Training & Test \\
\hline Accuracy (\%) & $94.9 \pm 0.3$ & $95.4 \pm 0.4$ & $94.0 \pm 0.8$ \\
Sensitivity (\%) & $93.9 \pm 0.6$ & $94.5 \pm 0.6$ & $92.8 \pm 1.7$ \\
Specificity (\%) & $95.6 \pm 0.6$ & $96.1 \pm 0.6$ & $94.8 \pm 1.4$ \\
Positive Predictive Value (\%) & $94.1 \pm 0.7$ & $94.7 \pm 0.8$ & $93.0 \pm 1.7$ \\
\hline \hline
\end{tabular}

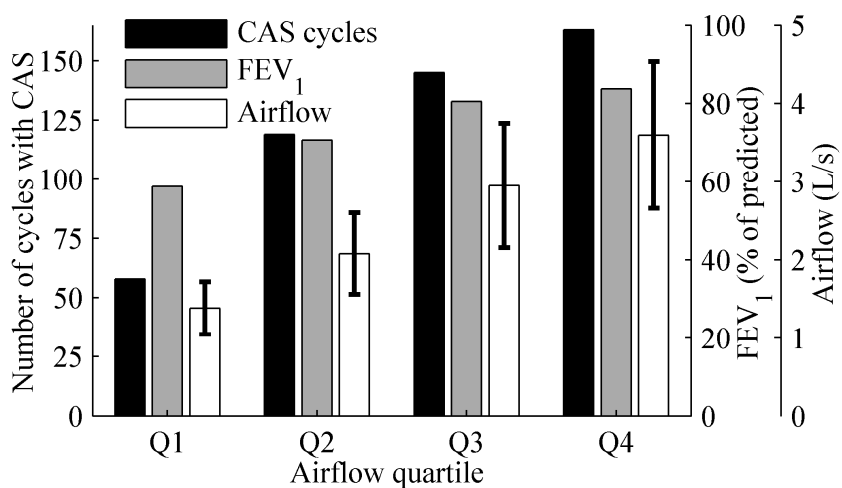

Fig. 7. Distribution of CAS cycles from the dataset among airflow quartiles (black). Mean $\mathrm{FEV}_{1}$ of patients who provided CAS cycles at each airflow quartile (grey). Mean and standard deviation of the airflow levels included in each airflow quartile (white).

results for the simulated CAS cycles described in the next Section III.B.

Furthermore, we evaluated the performance of our classifier for different airflow levels. Figure 7 shows the distribution of the CAS cycles from the dataset ( 485 cycles) among the four quartiles. Moreover, the mean $\mathrm{FEV}_{1}$ value was calculated from the asthmatic subjects who provided CAS in each quartile. As shown, cycles containing CAS not only appear at high airflows (Q3 and Q4), but also at low and moderate airflows (Q1 and Q2). It is noteworthy that patients who provided CAS at low airflows had lower $\mathrm{FEV}_{1}$ values than those who only provided CAS at high airflows. In fact, there is a direct relationship between the two variables. Patients with severe asthma (with low $\mathrm{FEV}_{1}$ values) may produce CAS as much at low airflows as at high airflows. However, those with mild asthma only generate CAS at high airflow levels. Independently of the airflow quartile, our classifier performed very well in all cases (see Table IV).

TABLE IV

CLASSIFICATION OF RECORDED RS FROM INSPIRATORY CYCLES

\begin{tabular}{lccccc}
\hline \hline \multicolumn{1}{c}{ TOTAL } & Total & Q1 & Q2 & Q3 & Q4 \\
\hline Accuracy (\%) & $\mathbf{9 4 . 6} \pm \mathbf{0 . 3}$ & $95.3 \pm 0.7$ & $94.5 \pm 0.6$ & $94.1 \pm 0.6$ & $94.7 \pm 0.6$ \\
Sensitivity (\%) & $\mathbf{9 4 . 2} \pm \mathbf{0 . 4}$ & $92.3 \pm 1.2$ & $94.7 \pm 0.5$ & $94.5 \pm 0.8$ & $94.4 \pm 0.5$ \\
Specificity (\%) & $\mathbf{9 5 . 0} \pm \mathbf{0 . 9}$ & $97.0 \pm 0.9$ & $94.2 \pm 1.2$ & $93.6 \pm 1.5$ & $95.4 \pm 1.6$ \\
Positive predictive value (\%) & $\mathbf{9 6 . 0} \pm \mathbf{0 . 7}$ & $94.4 \pm 1.6$ & $95.4 \pm 0.9$ & $95.1 \pm 1.1$ & $97.7 \pm 0.8$ \\
\hline \multicolumn{1}{c}{ TRAINING } & Total & Q1 & Q2 & Q3 & Q4 \\
\hline Accuracy (\%) & $\mathbf{9 5 . 0} \pm \mathbf{0 . 6}$ & $95.5 \pm 1.3$ & $94.8 \pm 1.3$ & $94.7 \pm 1.2$ & $95.2 \pm 1.1$ \\
Sensitivity (\%) & $\mathbf{9 4 . 6} \pm \mathbf{0 . 7}$ & $92.5 \pm 2.7$ & $94.9 \pm 1.4$ & $94.8 \pm 1.3$ & $94.9 \pm 1.3$ \\
Specificity (\%) & $\mathbf{9 5 . 6} \pm \mathbf{1 . 0}$ & $97.2 \pm 1.3$ & $94.7 \pm 1.9$ & $94.5 \pm 2.2$ & $96.0 \pm 2.4$ \\
Positive predictive value (\%) & $\mathbf{9 6 . 4} \pm \mathbf{0 . 8}$ & $94.9 \pm 2.3$ & $95.8 \pm 1.5$ & $95.8 \pm 1.6$ & $98.0 \pm 1.1$ \\
\hline \multicolumn{1}{c}{ TESTING } & Total & Q1 & Q2 & Q3 & Q4 \\
\hline Accuracy (\%) & $\mathbf{9 3 . 7} \pm \mathbf{1 . 2}$ & $94.9 \pm 2.3$ & $93.8 \pm 2.2$ & $92.9 \pm 2.1$ & $93.8 \pm 2.3$ \\
Sensitivity (\%) & $\mathbf{9 3 . 6} \pm \mathbf{1 . 9}$ & $91.9 \pm 5.1$ & $94.2 \pm 2.8$ & $93.8 \pm 3.1$ & $93.5 \pm 2.7$ \\
Specificity (\%) & $\mathbf{9 3 . 9} \pm \mathbf{2 . 0}$ & $96.5 \pm 2.9$ & $93.2 \pm 3.5$ & $91.8 \pm 4.1$ & $94.3 \pm 4.0$ \\
Positive predictive value (\%) & $\mathbf{9 5 . 1} \pm \mathbf{1 . 5}$ & $93.8 \pm 4.9$ & $94.7 \pm 2.6$ & $94.0 \pm 2.8$ & $97.2 \pm 1.9$ \\
\hline \hline
\end{tabular}




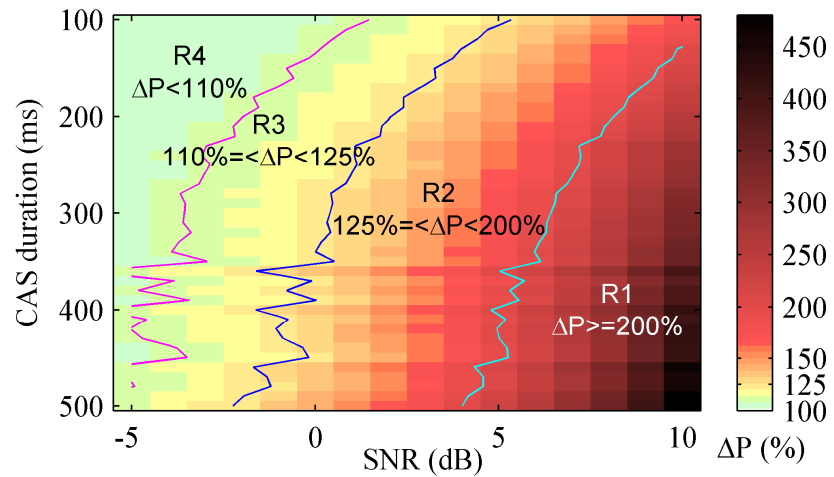

Fig. 8. Percentage increase of mean power $\left(\Delta \mathrm{P}_{\mathrm{ijk}}\right)$ of simulated CAS cycles, $w_{i j k}(t)$, with respect to their original normal sound cycles, $s_{k}(t)$, as a function of SNR and duration of CAS segments.

\section{B. Effect of SNR on Detection of Simulated CAS Cycles}

This section describes the results of the sensitivity analysis described in Section II.F.

Fig. 8 shows the results after obtaining 656 simulated CAS cycles from one inspiratory cycle with normal sounds, $s_{k}(t)$. The power increase $\left(\Delta P_{i j k}\right)$ parameter was used to evaluate the effect of adding CAS segments to an inspiratory cycle with normal sounds, with different durations and different SNRs. Three thresholds were set for power increases of $200 \%, 125 \%$, and $110 \%$, defining the boundaries for four regions of interest (R1-R4), plotted in Fig. 8.

The same procedure (described in Section II.F) was applied to six more inspiratory cycles with normal sounds, $s_{k}(t)$. Then, the classifier was applied to the total set of 4592 simulated CAS cycles. As shown in Fig. 9, the proposed classification algorithm achieved high accuracy for detecting cycles containing CAS in regions R1 $(98.7 \% \pm 1.4 \%)$ and R2 $(93.9 \%$ $\pm 4.1 \%$ ), while the accuracy was markedly lower in region $\mathrm{R} 4$ $(76.4 \% \pm 13.4 \%)$. However, region R4 corresponds to very low SNR values. This region included weak CAS, which had very low energy and only slightly increased the mean power of the RS signals $(\triangle P \leq 110 \%)$. Although there is no standard criterion for amplitude in the definition of CAS, considering the recorded CAS in the study dataset, regions R1-R2 represented more realistic SNR values. For this reason, the number of simulated CAS cycles progressively decreased from region $\mathrm{R} 1$ to region $\mathrm{R} 4$. In any case, the overall accuracy, including all regions, was $92.8 \% \pm 3.6 \%$. This high accuracy demonstrates that the proposed method offers high performance under both low and high SNR conditions. In fact, high accuracy $(87.7 \% \pm 7.3 \%)$ was achieved in region $\mathrm{R} 3$, which still represents an unfavorable scenario, in which the SNR may be below $0 \mathrm{~dB}$.

\section{Discussion AND CONCLUSIONS}

Asthma is characterized by a series of variable symptoms, including airflow limitation, shortness of breath, cough, and the presence of CAS [9]. Although these symptoms vary over time and CAS are not always present in asthma, when present, such sounds clearly indicate airway obstruction [4]. Therefore,

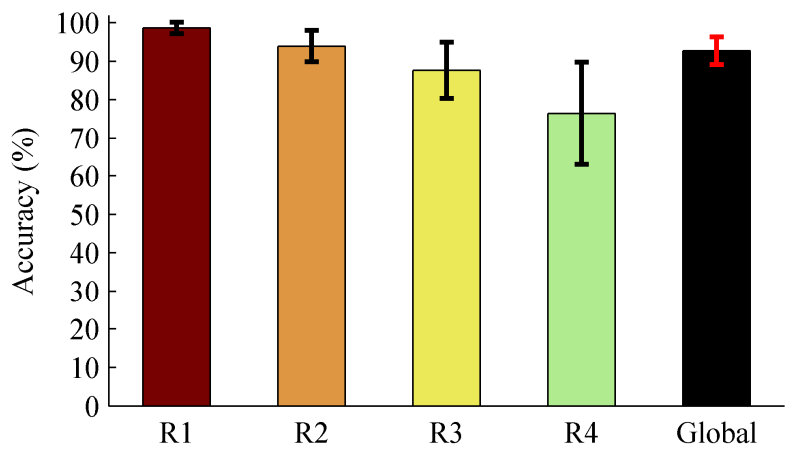

Fig. 9. Accuracy in simulated CAS cycle detection for regions R1-R4, which represent different SNR conditions (see Fig. 8).

detecting and analyzing CAS can provide some additional information about the pulmonary function of these patients.

This study demonstrates that the analysis of RS in terms of EEMD-based IFs provides an efficient, simple, and robust method for CAS detection and RS classification.

First, we have shown the viability of estimating the IF by EEMD. The definition of the IF of multi-component signals, such as RS, has been a subject of controversy, since strictly speaking the concept of IF is only meaningful for monocomponent signals. In this work, we found that EEMD allowed us to decompose RS into IMFs for which a physically meaningful IF was defined point-by-point. EEMD represented a key step in the multi-scale analysis of the IF in RS, as it determined the quality of the IF estimation and thus the performance of our RS classifier.

Indeed, we opted to use EEMD as it improves on the original EMD method, which had a mode mixing effect when applied to RS from some inspiratory cycles [33]. After comparing several methods addressing that mode mixing effect [34], [40][42], we identified some clear advantages of EEMD. The IMFs from EEMD had smaller frequency overlaps than IMFs from other methods. Due to properties similar to a dyadic filter bank, EEMD achieved a much better separation of different frequency scales, which reduced the mode mixing effect and improved the IF estimate. Moreover, thanks to the better separation of frequency scales, EEMD allowed us to analyze only IMFs 1-4 to cover the frequency range of interest, which goes from $100 \mathrm{~Hz}$ upwards for CAS detection. However, other methods required us to analyze at least 5 IMFs for the same purpose.

Two parameters determined the effectiveness of the EEMD: noise amplitude and number of iterations. If the added noise amplitude was too small (high SNR), there were few changes in the maxima of the original sound signal and, therefore, the benefits of EEMD were not evident. On the other hand, using high amplitude noise (low SNR) produced slightly noisy IMF components, but provided a reference scale distribution to enhance EMD and avoid the mode mixing effect. Furthermore, the residual noise level was able to be reduced by increasing the number of iterations. Indeed, a key issue was finding a balance between the SNR and number of iterations in order to minimize the mode mixing effect and obtain an acceptable residual noise level. 
As RS are random in nature, for this study, we added bandlimited noise to RS signals from inspiratory cycles at an SNR of $0 \mathrm{~dB}$, in order to perceive the benefits of EEMD. Despite this low SNR, applying 100 iterations to RS from each inspiratory cycle proved sufficient to obtain slightly noisy IMFs that allowed us to calculate a meaningful IF in a moderate time. Using fewer iterations would result in a higher residual noise level, whereas using more iterations would substantially increase the computation time. Furthermore, the slight residual noise was not a problem for our application, since we did not need a perfect decomposition to reconstruct the original signal from the IMFs, but rather we wanted to use them for IF estimates.

Although there are several methods of IF calculation [32], we found that the Hilbert method produced more accurate IF values than other methods, such as the Teager-Huang method, which produced more dispersed values.

We have presented a set of features, which were extracted from the IF and IE sequences, to train and test an SVM classifier for RS segments. For that purpose, a moving window was applied to the IF sequence from each IMF to calculate the dispersion, since we found that RS segments containing CAS were characterized by markedly lower IF dispersion [33]. We used the standard deviation just as a tool for measuring dispersion within each IF sequence. Then, we applied a set of thresholds (Fig. 3) to the IF dispersion in order to detect candidate CAS segments. Since these thresholds were proportional to the IF dispersion of each IMF, they adapted to the characteristics of each signal. The extracted IF dispersion and energy features (Fig. 5-b and 5-c) allowed us to achieve high sensitivity (94.2\%) and accuracy (94.6\%) in CAS detection and RS classification.

As shown in Table $\mathrm{V}$, the performance of our method is similar to or better than that reported for many previous CAS detection methods. However, these different approaches cannot be directly compared because there are some important differences between them, which affect the classification results. The main differences are in the following: the position and number of sensors (tracheal or lung sounds, monochannel or multichannel recordings), the respiratory maneuver performed (constant airflow, forced expiratory maneuver, or variable airflow), the respiratory disease of patients (asthma, COPD, or others), the size of the dataset, the types of CAS included in the dataset, or the parameters for evaluating the performance of the classifiers.

Despite the aforementioned differences, our approach has some objective advantages over previous approaches. We have shown the ability of our method to detect CAS derived from a wide range of airflow levels. Thanks to the progressive respiratory maneuver, which was performed by the asthmatic patients, the dataset of this study included CAS appearing at low airflows as well as that appearing at high airflows. This is a strength compared to previous studies for which RS were recorded at constant airflows or during forced expiratory maneuvers. We have shown that CAS may appear under different respiratory conditions, and that this has no effect on the performance of our classifier.
TABLE V

PERFORMANCE COMPARISON OF CAS DETECTION ALGORITHMS

\begin{tabular}{|c|c|c|c|}
\hline Features & Year & Method & Performance $(\%)$ \\
\hline EEMD + IF & 2014 & $\begin{array}{l}\text { Proposed } \\
\text { method }\end{array}$ & $\begin{array}{l}\text { Real: } 94.6 \text { (Acc), } \\
94.2(\mathrm{~S}), 95.0(\mathrm{Sp}) \\
\text { Sim: } 92.8 \text { (Acc) }\end{array}$ \\
\hline Spectrogram & 2004 & Homs [18] & $71<(S)<100$ \\
\hline Spectrogram & 2005 & Hsueh [24] & $89<(\mathrm{S}) \&(\mathrm{Sp})$ \\
\hline Spectrogram & 2006 & $\operatorname{Lin}[25]$ & $96.7(\mathrm{~S}), 90.9(\mathrm{Sp})$ \\
\hline Spectrogram & 2007 & Taplidou [19] & $95.5(\mathrm{~S}), 93.7(\mathrm{Sp})$ \\
\hline Spectrogram & 2008 & Jain [20] & $84(\mathrm{~S}), 86(\mathrm{Sp})$ \\
\hline Spectrogram & 2009 & Riella [21] & 84.8 (Acc) \\
\hline $\mathrm{MFCC}+\mathrm{GMM}$ & 2007 & Chien [14] & $90<($ Acc $)$ \\
\hline MFCC + GMM & 2009 & Bahoura [15] & $94.6(\mathrm{~S}), 91.9(\mathrm{Sp})$ \\
\hline Linear analysis & 2009 & Aydore [16] & 93.5 (Acc) \\
\hline Dominance spectrogram & 2011 & Jin [28] & 92.4 (Acc) \\
\hline $\mathrm{FT}+\mathrm{DWT}+\mathrm{PCA}$ & 2012 & Xie [12] & 97.3 (Acc) \\
\hline
\end{tabular}

Acc: accuracy, S: sensitivity, Sp: specificity, MFCC: Mel frequency cepstral coefficients, GMM: Gaussian mixture models, FT: Fourier transform, DWT: discrete wavelet transform, PCA: principal component analysis.

We have also performed a detailed sensitivity analysis of the influence of the SNR (amplitude of CAS) on the performance of the proposed classifier. We applied our classifier to simulated CAS cycles with different SNRs (see Fig. 8). As shown in Fig. 9, an overall accuracy of $92.8 \%$ was achieved. Although the performance of the RS classifier diminished from region $\mathrm{R} 1$ to $\mathrm{R} 4$, the accuracy was high $(87.7 \%$ in region $\mathrm{R} 3$ ) even at low SNRs. These results reinforce the reliability of our method, in that they demonstrate that this technique works properly under both high and low SNR conditions.

In contrast to previous approaches for RS analysis, such as those based on spectrograms or wavelet transformations, EEMD-IF used in the first stage of our classification scheme is an adaptive technique, which does not require a priori knowledge of the RS signal characteristics. That is, IF and IE sequences are calculated without choosing fixed analysis parameters and regardless of the type of RS and their temporal or spectral characteristics. On the other hand, IF and IE sequences allow us to work independently in either a timefrequency or a time-energy domain. For this reason, we were able to use dispersion-based criteria on IF sequences, this representing a novel and straightforward technique for detecting CAS segments. Moreover, IF and IE sequences are defined point-by-point, thus providing very high time resolution. In fact, EEMD-IF provides precise IF values, which represent the frequency content of RS signals at each time instant.

Thanks to the aforementioned properties of EEMD-IF, on which the proposed method relies, this is a suitable technique for RS classification, and also represents an alternative way of accurately analyzing RS signals. Consequently, unlike some techniques previously used for CAS detection, EEMD-IF could be used not only for detecting CAS, but also for characterizing these sounds. Although this proposed EEMD-IF technique has not been widely applied to RS analysis, various EMD-based approaches have already been used in previous studies either for detection and analysis of discontinuous adventitious sounds [43], [44], or for detection and separation of heart sounds from lung sounds [45]-[47]. 
In this study, we have focused on inspiratory cycles because inspiratory sounds are much louder than expiratory sounds on the back, where we recorded the analyzed RS. Nevertheless, this classifier could be effectively applied to RS from expiratory cycles and even from the full respiratory cycle. What is more, although this study is focused on RS from the surface of the back, the proposed classifier could be also applied to tracheal sounds. These factors do not have influence on the hypothesis of our method. That is, it depends on the variations of the IF dispersion inside a cycle, regardless of whether it is an inspiratory, an expiratory, or a full cycle.

The use of our RS classifier is a prior step for a detailed analysis of RS. Specifically, easier CAS detection will facilitate new research into the analysis and characterization of these sounds. On the other hand, the identification of normal inspiratory sounds, without CAS, is the basis for future analysis of normal inspiratory sound intensity. This classifier could be the first stage of a more complex system for the analysis of RS. Such a system could serve as much for the diagnosis of patients with obstructive respiratory diseases as for their long-term monitoring. The idea is that this type of system could be used in a routine way together with the spirometry. The combined information from these techniques could increase the reliability in the diagnostic assessment of these patients.

\section{ACKNOWLEDGMENT}

This work was made possible thanks to a collaboration agreement, between IBEC and IGTP, to create a joint research Unit. All authors would like to thank all the team in the Pulmonary Function Testing Laboratory at Germans Trias i Pujol University Hospital, for their collaboration in the patient recruitment as well as the performing of the spirometry tests.

\section{REFERENCES}

[1] A. Bohadana, G. Izbicki, and S.S. Kraman, "Fundamentals of lung auscultation," N. Engl. J. Med., vol. 370, pp. 744-751, Feb. 2014

[2] A.R.A. Sovijärvi, L.P. Malmberg, G. Charbonneau, J. Vanderschoot, F Dalmasso, C. Sacco, M. Rossi, and J.E. Earis, "Characteristics of breath sounds and adventitious respiratory sounds," Eur. Respir. Rev., vol. 10, pp. 591-596, 2000

[3] A.R.A. Sovijärvi, F. Dalmasso, J. Vanderschoot, L.P. Malmberg, G. Righini, and S.A.T. Stoneman, "Definitions of terms for applications of respiratory sounds," Eur. Respir. Rev., vol. 10, pp. 597-610, 2000.

[4] N. Meslier, G. Charbonneau, and J.L. Racineux, "Wheezes," Eur. Respir. J., vol. 8, pp. 1942-1948, Nov. 1995.

[5] J.J. Marini, D.J. Pierson, L.D. Hudson, and S. Lakshminarayan, "The significance of wheezing in chronic airflow obstruction," Am. Rev. Respir. Dis., vol. 120, pp. 1069-1072, Nov. 1979.

[6] J.A. Fiz, R. Jané, A. Homs, J. Izquierdo, M.A. García, and J. Morera, "Detection of wheezing during maximal forced exhalation in patients with obstructed airways," Chest, vol. 122, pp. 186-191, Jul. 2002.

[7] C.S. Shim and M.H. Williams, "Relationship of wheezing to the severity of obstruction in asthma," Arch. Intern. Med., vol. 143, pp. 890-892, May 1983

[8] J.A. Fiz, R. Jané, J. Izquierdo, A. Homs, M.A. García, R.Gómez, E. Monso, and J. Morera, "Analysis of forced wheezes in asthma patients," Respiration, vol. 73, pp. 55-60, Aug. 2006.

[9] E.D. Bateman et al., "Global strategy for asthma management and prevention: GINA executive summary," Eur. Respir. J., vol. 31, 143-78, Jan. 2008.
[10] A. Kandaswamy, C.S. Kumar, R.P. Ramanathan, S. Jayaraman, and N. Malmurugan, "Neural classification of lung sounds using wavelet coefficients," Comput. Biol. Med., vol. 34, pp. 523-537, Sep. 2004.

[11] A. Mondal, P. Bhattacharya, and G. Saha, "Detection of lungs status using morphological complexities of respiratory sounds," Sci. World J., vol. 2014, 9 pages, Feb. 2014.

[12] S. Xie, F. Jin, S. Krishnan, and F. Sattar, "Signal feature extraction by multi-scale PCA and its application to respiratory sound classification," Med. Biol. Eng. Comput., vol. 50, pp. 759-768, Jul. 2012.

[13] S. Matsunaga, K. Yamauchi, M. Yamashita, and S. Miyahara, "Classification between normal and abnormal respiratory sounds based on maximum likelihood approach," in Proc. IEEE Int. Conf. Acoust. Speech Signal Process., Taipei, 2009, pp. 517-520.

[14] J.C. Chien, H.D. Wu, F.C. Chong, and C.I. Li, "Wheeze detection using cepstral analysis in Gaussian mixture models," in Conf. Proc. IEEE Eng. Med. Biol. Soc., Lyon, 2007, pp. 3168-3171.

[15] M. Bahoura, "Pattern recognition methods applied to respiratory sounds classification into normal and wheeze classes," Comput. Biol. Med., vol. 39, pp. 824-843, Sep. 2009.

[16] S. Aydore, I. Sen, Y.P. Kahya, and M. Mihcak, "Classification of respiratory signals by linear analysis," in Conf. Proc. IEEE Eng. Med. Biol. Soc., Minneapolis, 2009, pp. 2617-2620.

[17] S.A. Taplidou, L.J. Hadjileontiadis, T. Penzel, V. Gross, and S.M. Panas, "WED: An efficient wheezing-episode detector based on breath sounds spectrogram analysis," in Conf. Proc. IEEE Eng. Med. Biol. Soc., Cancun, 2003, pp. 2531-2534.

[18] A. Homs-Corbera, J.A. Fiz, J. Morera, and R. Jané, “Time-frequency detection and analysis of wheezes during forced exhalation," IEEE Trans. Biomed. Eng., vol. 51, no. 1, pp. 182-186, Jan. 2004.

[19] S.A. Taplidou and L.J. Hadjileontiadis, "Wheeze detection based on time-frequency analysis of breath sounds," Comput. Biol. Med., vol. 37, pp. 1073-1083, Aug. 2007.

[20] A. Jain and J. Vepa, "Lung sound analysis for wheeze episode detection," in Conf. Proc. IEEE Eng. Med. Biol. Soc., Vancouver, 2008, pp. 25822585 .

[21] R.J. Riella, P. Nohama, and J.M. Maia, "Method for automatic detection of wheezing in lung sounds," Braz. J. Med. Biol. Res., vol. 42, pp. 674684, Jul. 2009.

[22] J. Zhang, W. Ser, J. Yu, and T.T. Zhang, "A novel wheeze detection method for wearable monitoring systems," in Int. Symp. Intell. Ubiquitous Comput. Educ., Chengdu, 2009, pp. 331-334.

[23] A. Oliveira, C. Pinho, J. Dinis, D. Oliveira, and A. Marques, "Automatic wheeze detection and lung function evaluation: a preliminary study," in Proc. Int. Conf. Health Inform., Barcelona, 2013, pp. 323-326.

[24] M.L. Hsueh, J.C. Chien, F.C. Chang, H.D. Wu, and F.C. Chong, "Respiratory wheeze detection system," in Conf. Proc. IEEE Eng. Med. Biol. Soc., Shanghai, 2005, pp. 7553-7559.

[25] B.S. Lin, B.S. Lin, H.D. Wu, F.C. Chong, and S.J. Chen, "Wheeze recognition based on 2D bilateral filtering of spectrogram," Biomed. Eng. Appl. Basis Commun., vol. 18, pp. 128-137, Jun. 2006.

[26] S.A. Taplidou et al., "On applying continuous wavelet transform in wheeze analysis," in Conf. Proc. IEEE Eng. Med. Biol. Soc., San Francisco, 2004, pp. 3832-3835.

[27] S.A. Taplidou and L.J. Hadjileontiadis, "Analysis of wheezes using wavelet higher order spectral features," IEEE Trans. Biomed. Eng., vol. 57, no. 7, pp. 1596-1610, Jul. 2010.

[28] F. Jin, S.S. Krishnan, and F. Sattar, "Adventitious sounds identification and extraction using temporal-spectral dominance-based features," IEEE Trans. Biomed. Eng., vol. 58, no. 11, pp. 3078-3087, Nov. 2011.

[29] N.E. Huang, Z. Shen, S.R. Long, M.C. Wu, H.H. Shih, Q. Zheng, N.C. Yen, C.C. Tung, and H.H. Liu, "The empirical mode decomposition and the Hilbert spectrum for nonlinear and non-stationary time series analysis," Proc. R. Soc. A, vol. 454, pp. 903-995, Mar. 1998.

[30] N.E. Huang, X. Chen, M.T. Lo, and Z. Wu, "On Hilbert spectral representation: a true time-frequency representation for nonlinear and nonstationary data," Adv. Adapt. Data Anal., vol. 3, pp. 63-93, Apr. 2011.

[31] B. Boashash, "Estimating and interpreting the instantaneous frequency of a signal-Part 1: Fundamentals," Proc. IEEE, vol. 80, no. 4, pp. 520538, Apr. 1992.

[32] N.E. Huang, Z. Wu, S.R. Long, K.C. Arnold, X. Chen, and K. Blank, "On instantaneous frequency," Adv. Adapt. Data Anal., vol. 1, pp. 177229, Apr. 2009. 
[33] M. Lozano, J.A. Fiz, and R. Jané, "Estimation of instantaneous frequency from empirical mode decomposition on respiratory sounds analysis," in Conf. Proc. IEEE Eng. Med. Biol. Soc., Osaka, 2013, pp. 981-984.

[34] Z. Wu and N.E. Huang, "Ensemble empirical mode decomposition: a noise-assisted data analysis method," Adv. Adapt. Data Anal., vol. 1, pp. 1-41, Jan. 2009.

[35] J. Zhang, R. Yan, R.X. Gao, and Z. Feng, "Performance enhancement of ensemble empirical mode decomposition," Mech. Syst. Signal Process., vol. 24, pp. 2104-2123, Oct. 2010.

[36] G. Rilling, P. Flandrin, and P. Gonçalvès, "On empirical mode decomposition and its algorithms," in Proc. IEEE/EURASIP Workshop Nonlinear Signal Image Process., Grado, 2003.

[37] http://perso.ens-lyon.fr/patrick.flandrin/emd.html

[38] D. Gabor, "Theory of communication," J. IEE-Part III: Radio Commun. Eng., vol. 93, no. 26, pp. 429-457, Nov. 1946.

[39] W.J. Tompkins, "Finite impulse response filters," in Biomedical Digital Signal Processing, New Jersey: Prentice-Hall, 1993, pp. 111-116.

[40] N. Rehman and D.P. Mandic, "Multivariate empirical mode decomposition," Proc. R. Soc. A, vol. 466, pp. 1291-1302, 2010.

[41] N. Rehman and D.P. Mandic, "Filter bank property of multivariate empirical mode decomposition," IEEE Trans. Signal Process., vol. 59, no. 5, pp. 2421-2426, May 2011.

[42] H. Hong, X. Wang, and Z. Tao, "Local integral mean-based sifting for empirical mode decomposition," IEEE Signal Process. Lett., vol. 16, no. 10, pp. 841-844, Oct. 2009.

[43] L.J. Hadjileontiadis, "Empirical mode decomposition and fractal dimension filter. A novel technique for denoising explosive lung sounds," IEEE Eng. Med. Biol. Mag., vol. 26, no. 1, pp. 30-39, 2007.

[44] B.A. Reyes, S. Charleston-Villalobos, R. Gonzalez-Camarena, and T. Aljama-Corrales, "Analysis of discontinuous adventitious lung sounds by Hilbert-Huang spectrum," in Conf. Proc. IEEE Eng. Med. Biol. Soc., Vancouver, 2008, pp. 3620-3623.

[45] S. Charleston-Villalobos, L.F. Dominguez-Robert, R. GonzalezCamarena, and A.T. Aljama-Corrales, "Heart sounds interference cancellation in lung sounds," in Conf. Proc. IEEE Eng. Med. Biol. Soc., New York, 2006, pp. 1694-1697.

[46] C. Papadaniil and L.J. Hadjileontiadis, "Efficient heart sound segmentation and extraction using ensemble empirical mode decomposition and kurtosis features," IEEE J. Biomed. Health Inform., vol. 18, no. 4, pp. 1138-1152, Jul. 2014.

[47] L. ChingShun, W.A. Tanumihardja, and S. HongHui, "Lung-heart sound separation using noise assisted multivariate empirical mode decomposition," in Int. Symp. Intell. Signal Process. Commun. Syst., Naha, 2013, pp. 726-730.

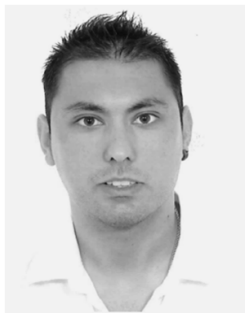

Manuel Lozano was born in Barcelona, Spain, in 1983. He received his B.Eng. degree in Telecommunications Engineering and M.S.E. in Biomedical Engineering from the Universitat Politècnica de Catalunya (UPC), Barcelona, Spain, in 2008 and 2011, respectively.
He is currently working towards a Ph.D. degree in Biomedical Engineering at UPC. Since 2011, he has been working as a research technician in the Innovation Mixed Unit at the Health Sciences Research Institute of the Germans Trias i Pujol Foundation (IGTP), Badalona, Spain, and the Institute for Bioengineering of Catalonia (IBEC), Barcelona, Spain. His research interests include biomedical signal processing and multichannel respiratory sound analysis in respiratory diseases.

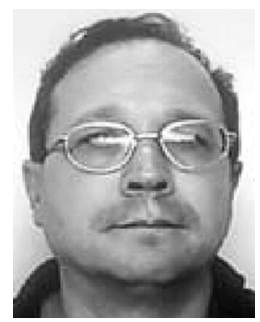

José Antonio Fiz was born in Pamplona, Spain, in 1953. He received his Diploma in Medicine from the University of Barcelona, Barcelona, Spain, in 1978. He specialized in Pulmonology at the University of Valencia and obtained his M.S.E. in Bioengineering from the UPC, Barcelona, Spain, in 1994.

Since 1991, he has been working in the respiratory physiology and sleep laboratories at the Germans Trias i Pujol University Hospital, Badalona, Spain. In 2008, he joined the IBEC and the Biomedical Research Networking center in Bioengineering, Biomaterials and Nanomedicine (CIBER-BBN). His research interests include respiratory sound analysis, sleep apnea, neural networks applied to clinical data, and clinical time series analysis.

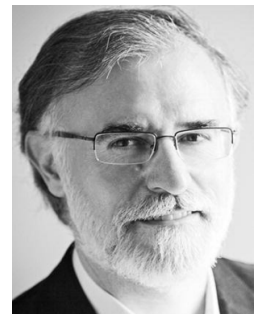

Raimon Jané (M'91, SM'14) received his Ph.D. from the UPC, Barcelona, Spain, in 1989.

He is currently Director of Research in the Department of Automatic Control (ESAII), UPC, and the Scientific Group Leader of the Biomedical Signal Processing and Interpretation Group, IBEC, Barcelona. Since 2008, he has been the Principal Investigator of the Biomedical Signals and Systems (SISBIO) Group of the CIBER-BBN. He is Professor of the Master's and the Coordinator of the Ph.D. programs in Biomedical Engineering. His research interests include multi-modal and multi-scale biomedical signal processing in cardiorespiratory and brain diseases.

Prof. Jané was a recipient of the Barcelona City Prize, from the Barcelona Council, in the area of technology research, in 2005. Currently, he is the President of the Spanish Society of Biomedical Engineering (SEIB). 